\title{
Bird Species Detection From Voice Features
}

\author{
Rachana B*, Kavya Hegde, Navya Bhat
}

Department of Computer Science, Srinivas Institute of Technology, Mangalore, Karnataka, India

\begin{tabular}{|c|c|}
\hline & ABSTRACT \\
\hline Article Info & The objective is naturally recognize which types of bird is available in a sound \\
\hline Volume 7 , Issue 4 & data set utilizing regulated learning. Contriving successful calculations for bird \\
\hline Page Number: 199-202 & $\begin{array}{l}\text { species order is a fundamental advance toward separating valuable natural } \\
\text { information from accounts gathered in the field. Here Naïve Bayes calculation to }\end{array}$ \\
\hline $\begin{array}{l}\text { Publication Issue : } \\
\text { July-August-2021 }\end{array}$ & $\begin{array}{l}\text { characterize bird voices into various species dependent on } 265 \text { highlights } \\
\text { removed from the chipping sound of birds. The difficulties in this undertaking } \\
\text { included memory the executives, the quantity of bird species for the machine }\end{array}$ \\
\hline Article History & perceive, and the jumble in signal-to-clamor proportion between the \\
\hline : 10 July 2021 & preparation and the testing sets. So to settle this difficulties we utilized Naïve \\
\hline \multirow[t]{3}{*}{ Published : 19 July 2021} & Bayes calculation from this we got great precision in it. The calculation Naive \\
\hline & Bayes got $91.58 \%$ exactness. \\
\hline & Keywords: Machine Learning, PC frameworks, Pseudo Code \\
\hline
\end{tabular}

\section{INTRODUCTION}

The mechanism of sound production from the vocal tract or vocalization in short, of each animal is different from another. Birds are numerous and easier to monitor than other species. There are many practical reasons behind the monitoring and locating a particular bird. First, ornithologists are always curious to locate a bird and study about it. Second, birds are endangered by different human activities like deforestation, poaching and overgrazing. Identification of a bird by its songs or calls can help in bird population census and thereby acts as an aid for conservation of bird species. Third, birds create security issue near airports. Hence identification of birds and their population census/control is important in present scenario.
This paper utilizes data set that contains bird tunes recorded in explicit geographic locale. Extraction of various highlights ordinarily found in solid order and discourse acknowledgment to get applicable qualities, and choice methodology for recognizable proof, where Machine Learning (ML) calculations are utilized to prepare utilized classifiers utilizing named data set of recently known types of revenue. Consequently recognize which types of bird is available in a sound account utilizing directed learning. Devising effective algorithms for bird species classification is a preliminary step toward the variety of voice collected. algorithms for bird species classification is a preliminary step toward the variety of voice collected. 


\section{METHODS AND MATERIAL}

Arrangement of this framework is finished utilizing Machine Learning approach. Machine Learning is logical investigation of calculation statical models that PC frameworks use to play out a particular assignment without utilizing express directions. Naive Bayes is a learning strategy for which a straightforward plan explicit property determination approach has shown great outcomes. Albeit this strategy manages arbitrary characteristics, it's the possibility to be misdirected when there are conditions among credits,and especially when repetitive ones are added. In any case,great outcomes have been accounted for utilizing the forward choice calculation which is better ready to identify when an excess property is going to be added than the regressive disposal approach related to a truly straightforward, nearly naïve metric that decides the normal of a characteristic subset to be essentially the exhibition of the learned calculation on the preparation

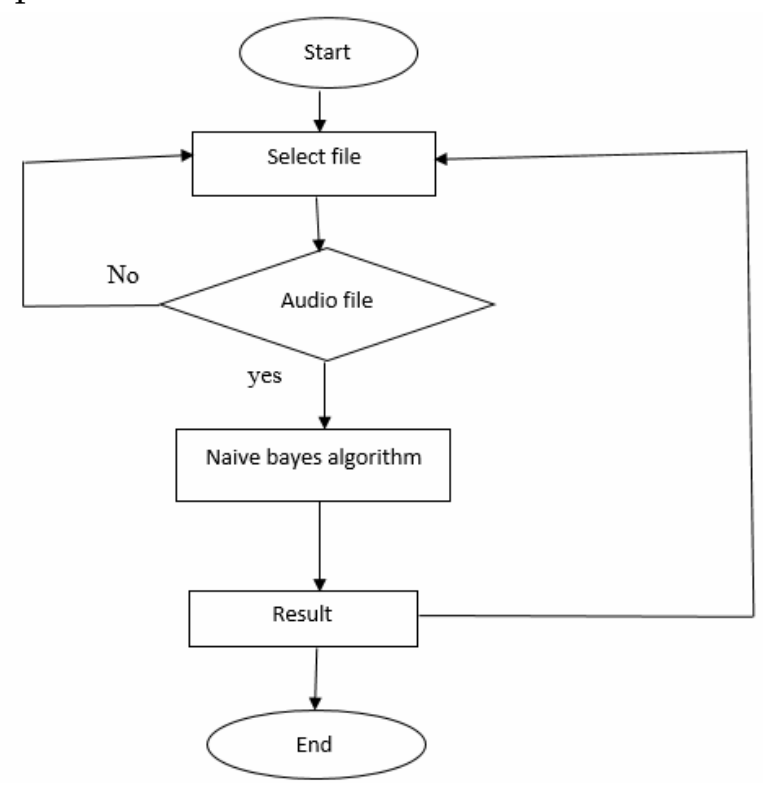

Fig.1 Flowchart for Bird species detection from voice features

Pseudo Code for Pre-processing and Feature Processing
Step 1: Read from read_csv

Step 2: Read audio file based on file_id

Step 3: Fetch sg, mask, data, audio_mask, sample_rate Step 4: Determine window size

Step 5: Extract features from each audio file For each audio frame get Species, genus, spec_centr_,

chromogram_ update weight file

Step 6: write weight file to external csv file

\section{Prediction}

Step 1: Read Input Audio File

Step 2 : Fetch sg, mask, data, SampleRate,

Audio_Marks

Step 3: Extract Features

Step 4: Import weight file

Step 5: Compare Model weights with Input audio features

Step 6: Displays Bird Species Name

\section{Procedure for Bird Voice Recognition in Non- \\ Realtime}

Step 1: Start

Step 2: Choose the audio file

Step 3: if button is equal to Naïve Bayes then Naïve Bayes algorithm is used for recognition.

Step 4: Bird Species displayed

Step 5: End

\section{RESULTS AND DISCUSSION}

- A genuine positive test outcome is one that identifies the condition when the condition is available.

- A genuine negative test outcome is one that doesn't distinguish the condition when the condition is missing.

- A positive test outcome is one that identifies the condition when the condition is missing. 
- A negative test outcome is one that doesn't distinguish the condition when the condition is available. Allow TP to mean the quantity of genuine positives, TN the quantity of genuine negatives, FP the quantity of bogus positives, and FN the quantity of bogus negatives.

- Sensitivity estimates the capacity of a test to identify the condition when the condition is available. Subsequently, Sensitivity = $\mathrm{TP} /(\mathrm{TP}+\mathrm{FN})$.

- $\quad$ Specificity estimates the capacity of a test to effectively bar the condition (not distinguish the condition) when the condition is missing. Along these lines, Specificity $=\mathrm{TN} /(\mathrm{TN}+\mathrm{FP})$.

- Predictive worth positive is the extent of positives that relate to the presence of the condition. Subsequently, Predictive worth positive $=\mathrm{TP} /(\mathrm{TP}+\mathrm{FP})$.

- Predictive worth negative is the extent of negatives that relate to the shortfall of the condition. Accordingly, Predictive worth negative $=\mathrm{TN} /(\mathrm{TN}+\mathrm{FN})$.

\section{TABLE I. CORRELATION TABLE}

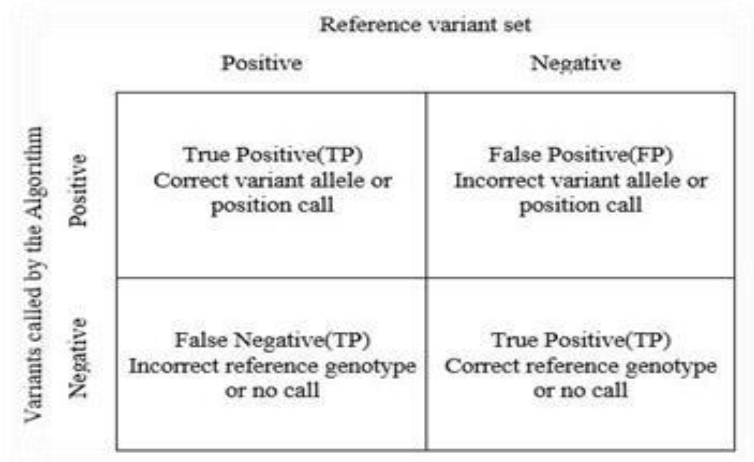

The training dataset consist of 265 bird audios. In non-real time the dataset consists of 265 bird's voices. It consists of both voice and noises then using Naïve Bayes algorithm it will separate both noises and bird voice. Then it will recognise the bird species.
TABLE II. RESULT ANALYSIS

\begin{tabular}{|l|l|l|l|l|l|l|}
\hline $\begin{array}{l}\text { Algorithm } \\
\text { Name }\end{array}$ & $\begin{array}{l}\text { No. of } \\
\text { Testing } \\
\text { audio }\end{array}$ & TP & TN & FP & FN & Result \\
\hline $\begin{array}{l}\text { Naïve } \\
\text { Bayes }\end{array}$ & 265 & 242 & 0 & 0 & 23 & 0.9158 \\
\hline
\end{tabular}

\section{CONCLUSION}

The Bird species detection is increasingly important role in markets. The application developed addresses the problem of manual detection of non-real time bird voices. The project demonstrates a novel Naïve Bayes method to recognize the bird species in nonreal. The experiments demonstrate that the Naïve Bayes obtained $91.58 \%$ classification accuracy in non-real-time. Using the audio file, then choose the algorithm and then we can find the bird species.

\section{REFERENCES}

[1]. Dorota kaminska, Artur Gmerek, "Automatic identification of bird species: A comparison between KNN and SOM classifiers," New trends in audio \& video/signal processing algorithms (NTAV/SPA), architectures, arrangements \& applications 27- 29th September, 2012.

[2]. Marcelo T. Lopes, Lucas L. Gioppo, Thiago T.Higushi, Celso A. A.Kaesther, Carlos N. Silla Jr., Alessandro L. koerich, "Automatic birdspecies identification for large number of species", IEEE International Symposium on Multimedia, 2011.

[3]. Jason Wimmer, Michael Towsey, Birgit Planitz, Ian Williamson, Paul Roe, "Analysing environmental acoustic data through collaboration and automation," Future Generation Computer Systems 29, 560-568, 2013 
[4]. Felix Weninger, Bjorn Schuller, "Audio recognition in the wild: static and dynamic classification on a real-world database of animal vocalizations," IEEE International Conference on Acoustics, Speech and Signal Processing (ICASSP), 2011.

[5]. Chang- Hsing Lee, Chih- Hsun chou, Chin chuan han, Ren Zhuang Huang, "Automatic recognition of animal vocalizations using averaged MFCC \& linear discriminant analysis," Pattern recognition letters 27(2006), 93-101 No. 1, pp.17-23, May 2006.

\section{Cite this article as :}

Rachana B, Kavya Hegde, Navya Bhat, "Bird Species Detection From Voice Features", International Journal of Scientific Research in Computer Science, Engineering and Information Technology (IJSRCSEIT), ISSN : 2456-3307, Volume 7, Issue 4, pp.199-202, July-August-2021. Available at doi : https://doi.org/10.32628/CSEIT217453 Journal URL : https://ijsrcseit.com/CSEIT217453 